\title{
Majorization inequalities via Green functions and Fink's identity with applications to Shannon entropy
}

\author{
Nouman Siddique ${ }^{1}$, Muhammad Imran ${ }^{1 *}$ (D), Khuram Ali Khan ${ }^{2}$ and Josip Pečarić ${ }^{3}$
}

\section{"Correspondence:}

drmimranchaudhry@gmail.com 1 Department of Mathematics, Government College University, 38000 Faisalabad, Pakistan

Full list of author information is available at the end of the article

\section{Springer}

\begin{abstract}
This paper is devoted to obtain generalized results related to majorization-type inequalities by using well-known Fink's identity and new types of Green functions, introduced by Mehmood et al. (J. Inequal. Appl. 2017:108, 2017). We give a generalized majorization theorem for the class of $n$-convex functions. We utilize the Csiszár f-divergence and generalized majorization-type inequalities in providing the corresponding generalizations. As an application, we present the obtained results in terms of Shannon entropy and Kullback-Leibler distance.
\end{abstract}

Keywords: Majorization inequailty; Fink's identity; New Green functions; $n$-convex functions; Čebyšev functional; Csiszár $f$-divergence; Kullback-Leibler divergence; Shannon entropy

\section{Introduction}

Majorization is a powerful and useful mathematical tool, which arises frequently in many different areas of research. Anyone seriously interested in majorization will eventually need to take a good look at Marshall et al's classic text [29], which gives a very nearly comprehensive coverage of both history and theory of majorization. It also contains a wealth of additional material including many interesting applications to diverse areas of mathematics. Alberti and Uhlmann [7] have also written a more specified monograph on majorization, which may be of interest to many readers. Moreover, Ando has written an excellent pair $[8,9]$ of survey papers on majorization, which provide a brief introduction to the subject and cover much of the field's development since the publication of Marshall et al.s book [29].

In 2018, Latif et al. [27] studied generalized results related to majorization inequality by using Taylor's polynomial in combination with newly introduced Green functions. In the same year, Siddique et al. [34] gave generalized majorization results via Lidstone's polynomial and newly defined Green functions. The theory of majorization often appears in many fields of applications. Khan et al. [20] presented significant material on majorization along with its applications in information theory.

(c) The Author(s) 2020. This article is licensed under a Creative Commons Attribution 4.0 International License, which permits use sharing, adaptation, distribution and reproduction in any medium or format, as long as you give appropriate credit to the original author(s) and the source, provide a link to the Creative Commons licence, and indicate if changes were made. The images or other third party material in this article are included in the article's Creative Commons licence, unless indicated otherwise in a credit line to the material. If material is not included in the article's Creative Commons licence and your intended use is not permitted by statutory regulation or exceeds the permitted use, you will need to obtain permission directly from the copyright holder. To view a copy of this licence, visit http://creativecommons.org/licenses/by/4.0/ 
In this paper, our main goal is obtaining generalized results about majorization by using new Green functions and Fink's identity. We further make connection of majorization with information theory and discuss our generalized majorization inequality in terms of divergences and entropies. The results we obtain in this paper are closely related to the contents of [1-5]. Moreover, some results related to the present topic can also be found in [19].

The following definition of majorization is from [33, p. 319].

Definition 1 For fixed $k \geq 2$, let $\mathbf{x}=\left(x_{1}, \ldots, x_{k}\right)$ and $\mathbf{y}=\left(y_{1}, \ldots, y_{k}\right)$ be two nonincreasing $k$-tuples of real numbers. If

$$
\sum_{i=1}^{j} y_{i} \leq \sum_{i=1}^{j} x_{i}
$$

for $j=1,2, \ldots, k-1$ and

$$
\sum_{i=1}^{k} x_{i}=\sum_{i=1}^{k} y_{i}
$$

then we say that $\mathbf{x}$ majorizes $\mathbf{y}$. Symbolically, we write $\mathbf{x} \succ \mathbf{y}$. Hardy et al. [15] introduced this notation and notion of majorization.

The following theorem is called the classical majorization theorem and is given in [29, p. 11] (see also [33, p. 320]).

Theorem A Consider two nonincreasing real k-tuples $\boldsymbol{x}=\left(x_{1}, \ldots, x_{k}\right)$ and $\boldsymbol{y}=\left(y_{1}, \ldots, y_{k}\right)$, where $x_{i}, y_{i} \in\left[\vartheta_{1}, \vartheta_{2}\right] \subset \mathbb{R}$ for $i=1, \ldots, k$. Then $\mathbf{x}$ majorizes $\mathbf{y}$ if and only if following inequality holds:

$$
\sum_{i=1}^{k} f\left(y_{i}\right) \leq \sum_{i=1}^{k} f\left(x_{i}\right),
$$

where $f:\left[\vartheta_{1}, \vartheta_{2}\right] \rightarrow \mathbb{R}$ is a continuous convex function.

The following theorem is regarded as a generalization of the majorization theorem given by Fuchs [14] (see also [33, p. 323] and [29, p. 580]).

Theorem B Consider two nonincreasing real k-tuples $\boldsymbol{x}=\left(x_{1}, \ldots, x_{k}\right)$ and $\boldsymbol{y}=\left(y_{1}, \ldots, y_{k}\right)$, where $x_{i}, y_{i} \in\left[\vartheta_{1}, \vartheta_{2}\right] \subset \mathbb{R}$ for $i=1, \ldots, k$. Let $\mathbf{p}=\left(p_{1}, \ldots, p_{k}\right)$ be a real $k$-tuple such that

$$
\sum_{i=1}^{j} p_{i} y_{i} \leq \sum_{i=1}^{j} p_{i} x_{i},
$$

for $j=1,2, \ldots, k-1$ and

$$
\sum_{i=1}^{k} p_{i} y_{i}=\sum_{i=1}^{k} p_{i} x_{i} .
$$


Then

$$
\sum_{i=1}^{k} p_{i} f\left(y_{i}\right) \leq \sum_{i=1}^{k} p_{i} f\left(x_{i}\right)
$$

where $f:\left[\vartheta_{1}, \vartheta_{2}\right] \rightarrow \mathbb{R}$ is a continuous convex function.

The following theorem represents an integral form of Theorem B and is in fact a simple consequence of Theorem 1 given in [31] (see also [33, p. 328].

Theorem C Let $\phi, \psi:[a, b] \rightarrow\left[\vartheta_{1}, \vartheta_{2}\right]$ be two continuous decreasing functions, and let $p:[a, b] \rightarrow \mathbb{R}$ be any continuous function. If

$$
\int_{a}^{\lambda} p(w) \psi(w) d w \leq \int_{a}^{\lambda} p(w) \phi(w) d w \quad \text { for every } \lambda \in[a, b]
$$

and

$$
\int_{a}^{b} p(w) \psi(w) d w=\int_{a}^{b} p(w) \phi(w) d w
$$

then

$$
\int_{a}^{b} p(w) f(\psi(w)) d w \leq \int_{a}^{b} p(w) f(\phi(w)) d w,
$$

where $f:\left[\vartheta_{1}, \vartheta_{2}\right] \rightarrow \mathbb{R}$ is a continuous convex function.

For other forms of integral version and generalization of the majorization theorem, see [29, p. 583], [10, 21, 23-26, 28]. In this paper, we present the results for decreasing functions $\phi$ and $\psi$ that satisfy the conditions of Theorem C, but those results also hold for increasing $\phi$ and $\psi$ satisfying the inequality

$$
\int_{\lambda}^{b} p(w) \psi(w) d w \leq \int_{\lambda}^{b} p(w) \phi(w) d w \quad \text { for every } \lambda \in[a, b]
$$

and condition (8). For instance, see the example in [29, p. 584].

In 1991, Fink [13] proved a very important result, known as Fink's identity, given as follows.

Theorem D Let $\left[\vartheta_{1}, \vartheta_{2}\right] \subset \mathbb{R}$ and $f:\left[\vartheta_{1}, \vartheta_{2}\right] \rightarrow \mathbb{R}, n \geq 1$, be such that $f^{(n-1)}$ is absolutely continuous. Then

$$
\begin{aligned}
f(x)= & \frac{n}{\vartheta_{2}-\vartheta_{1}} \int_{\vartheta_{1}}^{\vartheta_{2}} f(t) d t \\
& -\sum_{s=1}^{n-1}\left(\frac{n-s}{s !}\right)\left(\frac{f^{(s-1)}\left(\vartheta_{1}\right)\left(x-\vartheta_{1}\right)^{s}-f^{(s-1)}\left(\vartheta_{2}\right)\left(x-\vartheta_{2}\right)^{s}}{\vartheta_{2}-\vartheta_{1}}\right) \\
& +\frac{1}{(n-1) !\left(\vartheta_{2}-\vartheta_{1}\right)} \int_{\vartheta_{1}}^{\vartheta_{2}}(x-t)^{n-1} s^{\left[\vartheta_{1}, \vartheta_{2}\right]}(t, x) f^{(n)}(t) d t,
\end{aligned}
$$


where

$$
s^{\left[\vartheta_{1}, \vartheta_{2}\right]}(t, x)= \begin{cases}\left(t-\vartheta_{1}\right), & \vartheta_{1} \leq t \leq x \leq \vartheta_{2}, \\ \left(t-\vartheta_{2}\right), & \vartheta_{1} \leq x<t \leq \vartheta_{2} .\end{cases}
$$

As stated in [30], the complete reference about Abel-Gontscharoff polynomial and theorem for "two-point right focal problem" is given in [6].

Remark 1 The Abel-Gontscharoff polynomial, as a special choice of the "two-point right focal" interpolating polynomial for $n=2$, is

$$
f(u)=f\left(\vartheta_{1}\right)+\left(u-\vartheta_{1}\right) f^{\prime}\left(\vartheta_{2}\right)+\int_{\vartheta_{1}}^{\vartheta_{2}} G_{\Omega, 2}(u, v) f^{\prime \prime}(v) d v,
$$

where $G_{\Omega, 2}(u, v):\left[\vartheta_{1}, \vartheta_{2}\right] \times\left[\vartheta_{1}, \vartheta_{2}\right] \rightarrow \mathbb{R}$ is the Green function for the "two-point right focal problem" given as

$$
G_{1}(u, v)=G_{\Omega, 2}(u, v)= \begin{cases}\left(\vartheta_{1}-v\right), & \vartheta_{1} \leq v \leq u, \\ \left(\vartheta_{1}-u\right), & u \leq v \leq \vartheta_{2} .\end{cases}
$$

Let us define some new types of Green functions $G_{l}:\left[\vartheta_{1}, \vartheta_{2}\right] \times\left[\vartheta_{1}, \vartheta_{2}\right] \rightarrow \mathbb{R}$, where $\left[\vartheta_{1}, \vartheta_{2}\right] \subset \mathbb{R}$ and $l=2,3,4$, given by Mehmood et al. (see [30]), which are continuous and convex, by keeping in view the Abel-Gontscharoff Green's function for the "two-point right focal problem":

$$
\begin{aligned}
& G_{2}(u, v)= \begin{cases}\left(u-\vartheta_{2}\right), & \vartheta_{1} \leq v \leq u, \\
\left(v-\vartheta_{2}\right), & u \leq v \leq \vartheta_{2} ;\end{cases} \\
& G_{3}(u, v)= \begin{cases}\left(u-\vartheta_{1}\right), & \vartheta_{1} \leq v \leq u, \\
\left(v-\vartheta_{1}\right), & u \leq v \leq \vartheta_{2} ;\end{cases} \\
& G_{4}(u, v)= \begin{cases}\left(\vartheta_{2}-v\right), & \vartheta_{1} \leq v \leq u, \\
\left(\vartheta_{2}-u\right), & u \leq v \leq \vartheta_{2} .\end{cases}
\end{aligned}
$$

Next, we evaluate new generalizations of majorization inequality with the help of the following lemma given by Mehmood et al. [30].

Lemma 1 Let $f:\left[\vartheta_{1}, \vartheta_{2}\right] \rightarrow \mathbb{R}$ be a twice differentiable function, and let $G_{l}(l=1,2,3,4)$ be Green functions given in (14)-(17). Then along with identity (13), we have the following identities:

$$
\begin{aligned}
& f(u)=f\left(\vartheta_{2}\right)+\left(u-\vartheta_{2}\right) f^{\prime}\left(\vartheta_{1}\right)+\int_{\vartheta_{1}}^{\vartheta_{2}} G_{2}(u, v) f^{\prime \prime}(v) d v, \\
& f(u)=f\left(\vartheta_{2}\right)-\left(\vartheta_{2}-\vartheta_{1}\right) f^{\prime}\left(\vartheta_{2}\right)+\left(u-\vartheta_{1}\right) f^{\prime}\left(\vartheta_{1}\right)+\int_{\vartheta_{1}}^{\vartheta_{2}} G_{3}(u, v) f^{\prime \prime}(v) d v, \\
& f(u)=f\left(\vartheta_{1}\right)+\left(\vartheta_{2}-\vartheta_{1}\right) f^{\prime}\left(\vartheta_{1}\right)-\left(\vartheta_{2}-u\right) f^{\prime}\left(\vartheta_{2}\right)+\int_{\vartheta_{1}}^{\vartheta_{2}} G_{4}(u, v) f^{\prime \prime}(v) d v .
\end{aligned}
$$


This paper is arranged as follows. Section 2.1 contains some important results obtained by generalizing majorization-type identities by using Fink's identity and new Green functions. We also obtain a generalized majorization theorem for the class of $n$-convex functions. In Sect. 2.2, we use the Csiszár $f$-divergence and generalized majorization-type inequalities to obtain new generalized results. Further, we discuss our generalized results in terms of Shannon entropy and Kullback-Leibler distance.

\section{Main results}

This section contains results about the generalization of majorization inequality. For simplicity, we define some notations used in this paper. Let $\mathbf{x}=\left(x_{1}, \ldots, x_{k}\right), \mathbf{y}=\left(y_{1}, \ldots, y_{k}\right)$, and $\mathbf{p}=\left(p_{1}, \ldots, p_{k}\right)$ be such that $x_{i}, y_{i} \in\left[\vartheta_{1}, \vartheta_{2}\right] \subset \mathbb{R}$ and $p_{i} \in \mathbb{R}$ for $i=1,2, \ldots, k$. We denote the majorization difference for a function $f$ as

$$
\mathbb{D}(\mathbf{p}, \mathbf{x}, \mathbf{y}, f(\cdot)):=\sum_{i=1}^{k} p_{i} f\left(x_{i}\right)-\sum_{i=1}^{k} p_{i} f\left(y_{i}\right) .
$$

Let $p:[a, b] \rightarrow \mathbb{R}$ and $\phi, \psi:[a, b] \rightarrow\left[\vartheta_{1}, \vartheta_{2}\right]$ be continuous functions. We define the integral majorization difference for a function $f$ as

$$
\widetilde{\mathbb{D}}(p, \phi, \psi, f(\cdot)):=\int_{a}^{b} p(w)(f(\phi(w))-f(\psi(w))) d w .
$$

\subsection{Majorized results using Fink's identity}

First, we present two equivalent statements of majorization inequality between newly defined Green functions and continuous convex functions.

Theorem 1 Let $f:\left[\vartheta_{1}, \vartheta_{2}\right] \rightarrow \mathbb{R}$ be a continuous convex function, and let $\boldsymbol{x}=\left(x_{1}, \ldots, x_{k}\right)$, $\boldsymbol{y}=\left(y_{1}, \ldots, y_{k}\right)$, and $\boldsymbol{p}=\left(p_{1}, \ldots, p_{k}\right)$ be $k$-tuples such that $x_{i}, y_{i} \in\left[\vartheta_{1}, \vartheta_{2}\right]$ and $p_{i} \in \mathbb{R}$ for $i=$ $1,2, \ldots, k$ and condition (5) is satisfied. Let $G_{l}(l=1,2,3,4)$ be the Green functions given in (14)-(17). Then

$$
\sum_{i=1}^{k} p_{i} f\left(y_{i}\right) \leq \sum_{i=1}^{k} p_{i} f\left(x_{i}\right)
$$

if and only if

$$
\sum_{i=1}^{k} p_{i} G_{l}\left(y_{i}, v\right) \leq \sum_{i=1}^{k} p_{i} G_{l}\left(x_{i}, v\right)
$$

for all $v \in\left[\vartheta_{1}, \vartheta_{2}\right]$.

Proof Here we only give the proof for $l=4$, as the idea of the proof is the same for each $l=1,2,3,4$.

Let inequality (23) hold. Then $G_{4}(\cdot, v)\left(v \in\left[\vartheta_{1}, \vartheta_{2}\right]\right)$, being continuous and convex, satisfies inequality (23), that is, inequality (24) holds for $l=4$. 
Conversely, let $f$ be a convex function such that $f \in C^{2}\left(\left[\vartheta_{1}, \vartheta_{2}\right]\right)$ and (24) holds. Then by (20) we can write

$$
\begin{aligned}
& f\left(x_{i}\right)=f\left(\vartheta_{1}\right)+\left(\vartheta_{2}-\vartheta_{1}\right) f^{\prime}\left(\vartheta_{1}\right)-\left(\vartheta_{2}-x_{i}\right) f^{\prime}\left(\vartheta_{2}\right)+\int_{\vartheta_{1}}^{\vartheta_{2}} G_{4}\left(x_{i}, v\right) f^{\prime \prime}(v) d v, \\
& f\left(y_{i}\right)=f\left(\vartheta_{1}\right)+\left(\vartheta_{2}-\vartheta_{1}\right) f^{\prime}\left(\vartheta_{1}\right)-\left(\vartheta_{2}-y_{i}\right) f^{\prime}\left(\vartheta_{2}\right)+\int_{\vartheta_{1}}^{\vartheta_{2}} G_{4}\left(y_{i}, v\right) f^{\prime \prime}(v) d v .
\end{aligned}
$$

Using (25) and (26) along with (5), we have

$$
\sum_{i=1}^{k} p_{i} f\left(x_{i}\right)-\sum_{i=1}^{k} p_{i} f\left(y_{i}\right)=\int_{\vartheta_{1}}^{\vartheta_{2}}\left[\sum_{i=1}^{k} p_{i} G_{4}\left(x_{i}, v\right)-\sum_{i=1}^{k} p_{i} G_{4}\left(y_{i}, v\right)\right] f^{\prime \prime}(v) d v .
$$

Now the convexity of $f$ implies that $f^{\prime \prime}(v) \geq 0$ for all $v \in\left[\vartheta_{1}, \vartheta_{2}\right]$. Hence using (24) in (27), for $l=4$, we get (23).

Note that in this proof the requirement of the existence of the second derivative of $f$ is not necessary ([33], p. 172). We can directly eliminate this condition because it is possible to approximate uniformly continuous convex functions by convex polynomials.

Now we present some useful results obtained by generalizing majorization-type identities by using Fink's identity.

Theorem 2 Let $f:\left[\vartheta_{1}, \vartheta_{2}\right] \rightarrow \mathbb{R}$ be such that $f^{(n-1)}$ is absolutely continuous for $n \geq 3$. Let $\boldsymbol{x}=\left(x_{1}, \ldots, x_{k}\right), \boldsymbol{y}=\left(y_{1}, \ldots, y_{k}\right)$, and $\boldsymbol{p}=\left(p_{1}, \ldots, p_{k}\right)$ with $x_{i}, y_{i} \in\left[\vartheta_{1}, \vartheta_{2}\right] \subset \mathbb{R}$ and $p_{i} \in \mathbb{R}$ for $i=1,2, \ldots, k$. Also, let $s^{\left[\vartheta_{1}, \vartheta_{2}\right]}(t, x)$ be as defined in $(12)$, and let $G_{l}(l=1,2,3,4)$ be the Green functions given in (14)-(17). Then

$$
\begin{aligned}
\mathbb{D}(\mathbf{p}, \mathbf{x}, \mathbf{y}, f(\cdot)) \\
=f^{\prime}\left(\xi_{l}\right) \sum_{i=1}^{k} p_{i}\left(x_{i}-y_{i}\right)+\sum_{s=2}^{n-1}\left(\frac{n-s}{(s-2) !\left(\vartheta_{2}-\vartheta_{1}\right)}\right) \int_{\vartheta_{1}}^{\vartheta_{2}} \mathbb{D}\left(\mathbf{p}, \mathbf{x}, \mathbf{y}, G_{l}(\cdot, v)\right) \\
\quad \times\left(f^{(s-1)}\left(\vartheta_{2}\right)\left(v-\vartheta_{2}\right)^{s-2}-f^{(s-1)}\left(\vartheta_{1}\right)\left(v-\vartheta_{1}\right)^{s-2}\right) d v+\frac{1}{(n-3) !\left(\vartheta_{2}-\vartheta_{1}\right)} \\
\quad \times \int_{\vartheta_{1}}^{\vartheta_{2}} f^{(n)}(t)\left(\int_{\vartheta_{1}}^{\vartheta_{2}}\left(\mathbb{D}\left(\mathbf{p}, \mathbf{x}, \mathbf{y}, G_{l}(\cdot, v)\right)\right)(v-t)^{n-3} s^{\left[\vartheta_{1}, \vartheta_{2}\right]}(t, v) d v\right) d t,
\end{aligned}
$$

where $\xi_{1}, \xi_{4}=\vartheta_{2}$ and $\xi_{2}, \xi_{3}=\vartheta_{1}$.

Proof Here we only give the proof for $l=4$, as the idea of the proof is the same for each $l=1,2,3,4$.

Using (20) in the majorization difference (21), we get

$$
\mathbb{D}(\mathbf{p}, \mathbf{x}, \mathbf{y}, f(\cdot))=\sum_{i=1}^{k} p_{i}\left(x_{i}-y_{i}\right) f^{\prime}\left(\vartheta_{2}\right)+\int_{\vartheta_{1}}^{\vartheta_{2}} \mathbb{D}\left(\mathbf{p}, \mathbf{x}, \mathbf{y}, G_{4}(\cdot, v)\right) f^{\prime \prime}(\nu) d v .
$$


From Fink's identity (11) we have

$$
\begin{aligned}
f^{\prime \prime}(x)= & \sum_{s=2}^{n-1}\left(\frac{n-s}{(s-2) !}\right)\left(\frac{f^{(s-1)}\left(\vartheta_{2}\right)\left(x-\vartheta_{2}\right)^{s-2}-f^{(s-1)}\left(\vartheta_{1}\right)\left(x-\vartheta_{1}\right)^{s-2}}{\vartheta_{2}-\vartheta_{1}}\right) \\
& +\frac{1}{(n-3) !\left(\vartheta_{2}-\vartheta_{1}\right)} \int_{\vartheta_{1}}^{\vartheta_{2}}(x-t)^{n-3} s^{\left[\vartheta_{1}, \vartheta_{2}\right]}(t, x) f^{(n)}(t) d t .
\end{aligned}
$$

Using (30) in (29), we get

$$
\begin{aligned}
\mathbb{D}(\mathbf{p}, \mathbf{x}, \mathbf{y}, f(\cdot)) & \\
= & \sum_{i=1}^{k} p_{i}\left(x_{i}-y_{i}\right) f^{\prime}\left(\vartheta_{2}\right)+\int_{\vartheta_{1}}^{\vartheta_{2}} \mathbb{D}\left(\mathbf{p}, \mathbf{x}, \mathbf{y}, G_{4}(\cdot, v)\right) \sum_{s=2}^{n-1}\left(\frac{n-s}{(s-2) !}\right) \\
& \times\left(\frac{f^{(s-1)}\left(\vartheta_{2}\right)\left(v-\vartheta_{2}\right)^{s-2}-f^{(s-1)}\left(\vartheta_{1}\right)\left(v-\vartheta_{1}\right)^{s-2}}{\vartheta_{2}-\vartheta_{1}}\right) d v+\frac{1}{(n-3) !\left(\vartheta_{2}-\vartheta_{1}\right)} \\
& \times \int_{\vartheta_{1}}^{\vartheta_{2}} \mathbb{D}\left(\mathbf{p}, \mathbf{x}, \mathbf{y}, G_{4}(\cdot, v)\right)\left(\int_{\vartheta_{1}}^{\vartheta_{2}}(v-t)^{n-3} s^{\left[\vartheta_{1}, \vartheta_{2}\right]}(t, v) f^{(n)}(t) d t\right) d v .
\end{aligned}
$$

Rearranging (31) and applying Fubini's theorem in the last term, we obtain (28) for $l=4$.

An integral version of Theorem 2 is as follows.

Theorem 3 Let $\left[\vartheta_{1}, \vartheta_{2}\right] \subset \mathbb{R}$, and let $f:\left[\vartheta_{1}, \vartheta_{2}\right] \rightarrow \mathbb{R}$ be such that $f^{(n-1)}$ is absolutely continuous for $n \geq 3$. Let $p:[a, b] \rightarrow \mathbb{R}$ and $\phi, \psi:[a, b] \rightarrow\left[\vartheta_{1}, \vartheta_{2}\right]$ be continuous functions. Also, let $s^{\left[\vartheta_{1}, \vartheta_{2}\right]}(t, x)$ be as defined in $(12)$, and let $G_{l}(l=1,2,3,4)$ be the Green functions given in (14)-(17). Then

$$
\begin{aligned}
\widetilde{\mathbb{D}}(p, \phi, \psi, f(\cdot)) \\
=f^{\prime}\left(\xi_{l}\right) \int_{a}^{b} p(w)(\phi(w)-\psi(w)) d w+\sum_{s=2}^{n-1}\left(\frac{n-s}{(s-2) !\left(\vartheta_{2}-\vartheta_{1}\right)}\right) \\
\quad \times \int_{\vartheta_{1}}^{\vartheta_{2}} \tilde{\mathbb{D}}\left(p, \phi, \psi, G_{l}(\cdot, v)\right)\left(f^{(s-1)}\left(\vartheta_{2}\right)\left(v-\vartheta_{2}\right)^{s-2}-f^{(s-1)}\left(\vartheta_{1}\right)\left(v-\vartheta_{1}\right)^{s-2}\right) d v \\
\quad+\frac{1}{(n-3) !\left(\vartheta_{2}-\vartheta_{1}\right)} \\
\quad \times \int_{\vartheta_{1}}^{\vartheta_{2}} f^{(n)}(t)\left(\int_{\vartheta_{1}}^{\vartheta_{2}} \widetilde{\mathbb{D}}\left(p, \phi, \psi, G_{l}(\cdot, v)\right)(v-t)^{n-3} s^{\left[\vartheta_{1}, \vartheta_{2}\right]}(t, v) d v\right) d t,
\end{aligned}
$$

where $\xi_{1}, \xi_{4}=\vartheta_{2}$ and $\xi_{2}, \xi_{3}=\vartheta_{1}$.

Proof Using (20) in the integral majorization difference (22) and performing similar steps as in the proof of Theorem 2, we get the desired result for $l=4$. Similarly, the result can be proved for $l=1,2,3$. 
Theorem 4 Let all the assumptions of Theorem 2 hold, and let $f$ be an n-convex function. If

$$
\int_{\vartheta_{1}}^{\vartheta_{2}} \mathbb{D}\left(\mathbf{p}, \mathbf{x}, \mathbf{y}, G_{l}(\cdot, v)\right)(v-t)^{n-3} s^{\left[\vartheta_{1}, \vartheta_{2}\right]}(t, v) d v \geq 0,
$$

then

$$
\begin{aligned}
\mathbb{D}(\mathbf{p}, \mathbf{x}, \mathbf{y}, f(\cdot)) \geq & f^{\prime}\left(\xi_{l}\right) \sum_{i=1}^{k} p_{i}\left(x_{i}-y_{i}\right)+\sum_{s=2}^{n-1}\left(\frac{n-s}{(s-2) !\left(\vartheta_{2}-\vartheta_{1}\right)}\right) \int_{\vartheta_{1}}^{\vartheta_{2}} \mathbb{D}\left(\mathbf{p}, \mathbf{x}, \mathbf{y}, G_{l}(\cdot, v)\right) \\
& \times\left(f^{(s-1)}\left(\vartheta_{2}\right)\left(v-\vartheta_{2}\right)^{s-2}-f^{(s-1)}\left(\vartheta_{1}\right)\left(v-\vartheta_{1}\right)^{s-2}\right) d v,
\end{aligned}
$$

where $\xi_{1}, \xi_{4}=\vartheta_{2}$ and $\xi_{2}, \xi_{3}=\vartheta_{1}$.

Moreover, (34) holds in the reverse direction if inequality (33) is reversed.

Proof Since $f$ is an $n$-convex function, we have $f^{(n)}(x) \geq 0$ for all $x \in\left[\vartheta_{1}, \vartheta_{2}\right]$. Therefore using (33) in (28), we have (34). Moreover, if inequality (33) is reversed, then using (28), we obtain (34) in the reverse direction.

Theorem 5 Let all the assumptions of Theorem 3 be satisfied with $n$-convex $f$. If

$$
\int_{\vartheta_{1}}^{\vartheta_{2}} \widetilde{\mathbb{D}}\left(p, \phi, \psi, G_{l}(\cdot, v)\right)(v-t)^{n-3} s^{\left[\vartheta_{1}, \vartheta_{2}\right]}(t, v) d v \geq 0,
$$

then

$$
\begin{aligned}
& \widetilde{\mathbb{D}}(p, \phi, \psi, f(\cdot)) \\
& \quad \geq f^{\prime}\left(\xi_{l}\right) \int_{a}^{b} p(w)(\phi(w)-\psi(w)) d w+\sum_{s=2}^{n-1}\left(\frac{n-s}{(s-2) !\left(\vartheta_{2}-\vartheta_{1}\right)}\right) \\
& \quad \times \int_{\vartheta_{1}}^{\vartheta_{2}} \tilde{\mathbb{D}}\left(p, \phi, \psi, G_{l}(\cdot, v)\right)\left(f^{(s-1)}\left(\vartheta_{2}\right)\left(v-\vartheta_{2}\right)^{s-2}-f^{(s-1)}\left(\vartheta_{1}\right)\left(v-\vartheta_{1}\right)^{s-2}\right) d v,
\end{aligned}
$$

where $\xi_{1}, \xi_{4}=\vartheta_{2}$ and $\xi_{2}, \xi_{3}=\vartheta_{1}$.

Moreover, (36) holds in the reverse direction if inequality (35) is reversed.

Proof Using (35) in (32) and performing similar steps as in the proof of Theorem 4, we get required result.

A refinement of the weighted majorization-type inequality for two nonincreasing real $k$-tuples is given in the following corollary.

Corollary 1 Let all the assumptions of Theorem 2 be satisfied. Let $\boldsymbol{x}=\left(x_{1}, \ldots, x_{k}\right)$ and $\boldsymbol{y}=$ $\left(y_{1}, \ldots, y_{k}\right)$ be two nonincreasing real $k$-tuples such that (4) and (5) hold. 
(i) Let $f:\left[\vartheta_{1}, \vartheta_{2}\right] \rightarrow \mathbb{R}$ is an $n$-convex function with even $n>3$. Then

$$
\begin{aligned}
\mathbb{D}(\mathbf{p}, \mathbf{x}, \mathbf{y}, f(\cdot)) \geq & \sum_{s=2}^{n-1}\left(\frac{n-s}{(s-2) !\left(\vartheta_{2}-\vartheta_{1}\right)}\right) \int_{\vartheta_{1}}^{\vartheta_{2}} \mathbb{D}\left(\mathbf{p}, \mathbf{x}, \mathbf{y}, G_{l}(\cdot, v)\right) \\
& \times\left(f^{(s-1)}\left(\vartheta_{2}\right)\left(v-\vartheta_{2}\right)^{s-2}-f^{(s-1)}\left(\vartheta_{1}\right)\left(v-\vartheta_{1}\right)^{s-2}\right) d v .
\end{aligned}
$$

(ii) If inequality (37) holds and the function $\varphi:\left[\vartheta_{1}, \vartheta_{2}\right] \rightarrow \mathbb{R}$ defined by

$$
\begin{aligned}
\varphi(\cdot)= & \sum_{s=2}^{n-1}\left(\frac{n-s}{(s-2) !\left(\vartheta_{2}-\vartheta_{1}\right)}\right) \int_{\vartheta_{1}}^{\vartheta_{2}} G_{l}(\cdot, v) \\
& \times\left(f^{(s-1)}\left(\vartheta_{2}\right)\left(v-\vartheta_{2}\right)^{s-2}-f^{(s-1)}\left(\vartheta_{1}\right)\left(v-\vartheta_{1}\right)^{s-2}\right) d v
\end{aligned}
$$

is convex, then the right-hand side of (37) is nonnegative, that is, (6) is satisfied.

Proof (i): Since $G_{l}$ is continuous and convex for $l=1,2,3,4$, by Theorem B we can write

$$
\mathbb{D}\left(\mathbf{p}, \mathbf{x}, \mathbf{y}, G_{l}(\cdot, v)\right) \geq 0 \text {. }
$$

Note that for $\vartheta_{1} \leq v \leq t$, if $n>3$ is even, then we have

$$
\int_{\vartheta_{1}}^{t} \mathbb{D}\left(\mathbf{p}, \mathbf{x}, \mathbf{y}, G_{l}(\cdot, v)\right)(v-t)^{n-3} s^{\left[\vartheta_{1}, \vartheta_{2}\right]}(t, v) d v \geq 0,
$$

and for $t \leq v \leq \vartheta_{2}$ and even $n>3$, we have

$$
\int_{t}^{\vartheta_{2}} \mathbb{D}\left(\mathbf{p}, \mathbf{x}, \mathbf{y}, G_{l}(\cdot, v)\right)(v-t)^{n-3} s^{\left[\vartheta_{1}, \vartheta_{2}\right]}(t, v) d v \geq 0 .
$$

Combining (40) and (41), we get (33) for even $n>3$. Also, $f$ is $n$-convex for even $n>3$, so using Theorem 4 and (5), we have (37).

(ii): Clearly, we can write (37) in the following form:

$$
\mathbb{D}(\mathbf{p}, \mathbf{x}, \mathbf{y}, f(\cdot)) \geq \mathbb{D}(\mathbf{p}, \mathbf{x}, \mathbf{y}, \varphi(\cdot)) .
$$

Now the use of the convex function $\varphi$ in (6) leads us to the nonnegativity of the right-hand side of (42), which gives the required result.

A refinement of the integral majorization-type inequality for two decreasing funtions $\phi$ and $\psi$ is given in the following corollary, which is in fact the integral version of Corollary 1.

Corollary 2 Let all the assumptions of Theorem 3 hold. Let $\phi$ and $\psi$ be two decreasing functions such that (7) and (8) are satisfied.

(i) Iff $:\left[\vartheta_{1}, \vartheta_{2}\right] \rightarrow \mathbb{R}$ is an $n$-convex function, where $n>3$ is even, then

$$
\begin{aligned}
\widetilde{\mathbb{D}}(p, \phi, \psi, f(\cdot)) \geq & \sum_{s=2}^{n-1}\left(\frac{n-s}{(s-2) !\left(\vartheta_{2}-\vartheta_{1}\right)}\right) \int_{\vartheta_{1}}^{\vartheta_{2}} \widetilde{\mathbb{D}}\left(p, \phi, \psi, G_{l}(\cdot, v)\right) \\
& \times\left(f^{(s-1)}\left(\vartheta_{2}\right)\left(v-\vartheta_{2}\right)^{s-2}-f^{(s-1)}\left(\vartheta_{1}\right)\left(v-\vartheta_{1}\right)^{s-2}\right) d \nu .
\end{aligned}
$$


(ii) If inequality (43) holds and the function $\widetilde{\varphi}$ defined by

$$
\begin{aligned}
\widetilde{\varphi}(\cdot)= & \sum_{s=2}^{n-1}\left(\frac{n-s}{(s-2) !\left(\vartheta_{2}-\vartheta_{1}\right)}\right) \int_{\vartheta_{1}}^{\vartheta_{2}} G_{l}(\cdot, v) \\
& \times\left(f^{(s-1)}\left(\vartheta_{2}\right)\left(v-\vartheta_{2}\right)^{s-2}-f^{(s-1)}\left(\vartheta_{1}\right)\left(v-\vartheta_{1}\right)^{s-2}\right) d v
\end{aligned}
$$

is convex, then the right-hand side of (43) is nonnegative, that is, (9) is satisfied.

Proof Similar to the proof of Corollary 1.

\section{Remarks 1}

(i) We can give upper bounds like Grüss- and Ostrowski-type inequalities for our generalized identities. We can also obtain Lagrange- and Cauchy-type mean value theorems by using linear functionals deduced from our generalized results (see, e.g., $[27,32,34])$.

(ii) We can use an elegant method introduced by Jakšetić and Pečarć $[17,18]$ (see also $[22,30])$ to obtain $n$-exponential convexity, exponential convexity, and log-convexity with the help of linear functionals deduced from our generalized results on a given family with the same property for both discrete and integral cases. For more detail, see [32].

\subsection{Csiszár f-divergence for majorization}

In this section, we investigate generalized majorization-type inequality (37) in terms of divergences and entropies. We use Csiszár $f$-divergence and generalized majorization-type inequalities to obtain new generalized results. Moreover, we also discuss results related to Shannon entropy and Kullback-Leibler (K-L) distance.

The notion of $f$-divergence is introduced by Csiszár in [11]. For details, see [12].

Definition 2 Let $f: \mathbb{R}_{+} \rightarrow \mathbb{R}_{+}$be a convex function. If $\mathbf{r}=\left(r_{1}, \ldots, r_{k}\right)$ and $\mathbf{w}=\left(w_{1}, \ldots, w_{k}\right)$ are two positive probability distributions, then the $f$-divergence functional is

$$
I_{f}(\mathbf{r}, \mathbf{w}):=\sum_{i=1}^{k} w_{i} f\left(\frac{r_{i}}{w_{i}}\right) .
$$

Note that in the $f$-divergence functional, nonnegative probability distributions can also be used by defining

$$
f(0):=\lim _{t \rightarrow 0^{+}} f(t) ; \quad 0 f\left(\frac{0}{0}\right):=0 ; \quad 0 f\left(\frac{a}{t}\right):=0, \quad a>0 .
$$

Horváth et al. [16] considered the following functionality based on the previous definition.

Definition 3 Let $J \subset \mathbb{R}$ be an interval, and let $f: J \rightarrow \mathbb{R}$ be an $n$-convex function. Let $\mathbf{r}=\left(r_{1}, \ldots, r_{k}\right)$ be a real $\mathrm{k}$-tuple, and let $\mathbf{w}=\left(w_{1}, \ldots, w_{k}\right)$ be a positive real $\mathrm{k}$-tuple such that $\frac{r_{i}}{w_{i}} \in J, i=1,2, \ldots, k$. Then

$$
\widetilde{I}_{f}(\mathbf{r}, \mathbf{w}):=\sum_{i=1}^{k} w_{i} f\left(\frac{r_{i}}{w_{i}}\right) .
$$


Let $\mathbf{r}=\left(r_{1}, \ldots, r_{k}\right)$ and $\mathbf{w}=\left(w_{1}, \ldots, w_{k}\right)$ be two $k$-tuples. We use the following notation:

$$
\frac{\mathbf{r}}{\mathbf{w}}:=\left(\frac{r_{1}}{w_{1}}, \frac{r_{2}}{w_{2}}, \ldots, \frac{r_{k}}{w_{k}}\right) \quad \text { and } \quad \widetilde{I}_{G_{l}}(\mathbf{r}, \mathbf{w}, v):=\sum_{i=1}^{k} w_{i} G_{l}\left(\frac{r_{i}}{w_{i}}, v\right)
$$

The following theorem connects the generalized majorization-type inequality given in Corollary 1 and the Csiszár $f$-divergence.

Theorem 6 Let $f:\left[\vartheta_{1}, \vartheta_{2}\right] \rightarrow \mathbb{R}$ be such that $f^{(n-1)}$ is absolutely continuous for $n>3$. Also, let $s^{\left[\vartheta_{1}, \vartheta_{2}\right]}(t, x)$ be as defined in (12), and let $G_{l}(l=1,2,3,4)$ be the Green functions defined in (14)-(17). Also, let $\boldsymbol{q}=\left(q_{1}, \ldots, q_{k}\right), \boldsymbol{r}=\left(r_{1}, \ldots, r_{k}\right)$, and $\boldsymbol{w}=\left(w_{1}, \ldots, w_{k}\right)$ with $q_{i}, r_{i} \in \mathbb{R}$ and $w_{i} \in \mathbb{R}_{+}$for $i=1,2, \ldots, k$. Let

$$
\sum_{i=1}^{j} r_{i} \leq \sum_{i=1}^{j} q_{i}
$$

for $j=1,2, \ldots, k-1$ and

$$
\sum_{i=1}^{k} r_{i}=\sum_{i=1}^{k} q_{i}
$$

with $\frac{q_{i}}{w_{i}}, \frac{r_{i}}{w_{i}} \in\left[\vartheta_{1}, \vartheta_{2}\right](i=1,2, \ldots, k)$.If $\frac{q}{w}$ and $\frac{r}{w}$ are decreasing and $f$ is an $n$-convex function for even $n>3$, then

$$
\begin{aligned}
\widetilde{I}_{f}(\boldsymbol{q}, \boldsymbol{w}) \geq & \widetilde{I}_{f}(\boldsymbol{r}, \boldsymbol{w})+\sum_{s=2}^{n-1}\left(\frac{n-s}{(s-2) !\left(\vartheta_{2}-\vartheta_{1}\right)}\right) \int_{\vartheta_{1}}^{\vartheta_{2}}\left(\widetilde{I}_{G_{l}}(\boldsymbol{q}, \boldsymbol{w}, v)-\widetilde{I}_{G_{l}}(\boldsymbol{r}, \boldsymbol{w}, v)\right) \\
& \times\left(f^{(s-1)}\left(\vartheta_{2}\right)\left(v-\vartheta_{2}\right)^{s-2}-f^{(s-1)}\left(\vartheta_{1}\right)\left(v-\vartheta_{1}\right)^{s-2}\right) d v .
\end{aligned}
$$

Proof Take $x_{i}=\frac{q_{i}}{w_{i}}, y_{i}=\frac{r_{i}}{w_{i}}$, and $p_{i}=w_{i}>0(i=1,2, \ldots, k)$. Then conditions (45) and (46) imply conditions (4) and (5). So using these substitutions in (37), we get (47).

Theorem 7 Let $g:\left[\vartheta_{1}, \vartheta_{2}\right] \rightarrow \mathbb{R}$ be a function. Iffor $f(x):=x g(x), x \in\left[\vartheta_{1}, \vartheta_{2}\right]$, all conditions of Theorem 6 hold, then

$$
\begin{aligned}
\widehat{I}_{g}(\boldsymbol{q}, \boldsymbol{w}):= & \sum_{i=1}^{k} q_{i} g\left(\frac{q_{i}}{w_{i}}\right) \geq \widehat{I}_{g}(\boldsymbol{r}, \boldsymbol{w})+\sum_{s=2}^{n-1}\left(\frac{n-s}{(s-2) !\left(\vartheta_{2}-\vartheta_{1}\right)}\right) \\
& \times \int_{\vartheta_{1}}^{\vartheta_{2}}\left(\widetilde{I}_{G_{l}}(\boldsymbol{q}, \boldsymbol{w}, v)-\widetilde{I}_{G_{l}}(\boldsymbol{r}, \boldsymbol{w}, v)\right)\left(\frac{(x g)^{(s-1)}\left(\vartheta_{2}\right)}{\left(v-\vartheta_{2}\right)^{2-s}}-\frac{(x g)^{(s-1)}\left(\vartheta_{1}\right)}{\left(v-\vartheta_{1}\right)^{2-s}}\right) d v .
\end{aligned}
$$

Proof Following the proof of Theorem 6 for $f(x):=x g(x)$, we get (48).

The notion of entropic measure of disorder and the theory of majorization are closely related. Next, we present two special cases for majorization relations with connection to entropic inequalities.

In the first case, we discuss generalized majorization-type inequality with the entropy of a discrete probability distribution. 
Definition 4 Let $\mathbf{r}=\left(r_{1}, \ldots, r_{k}\right)$ be a positive probability distribution. Then the Shannon entropy of $\mathbf{r}$ is defined as

$$
H(\mathbf{r}):=-\sum_{i=1}^{k} r_{i} \log r_{i} .
$$

Note that the definition does not provide any problem for zero probability case, because $\lim _{x \rightarrow 0} x \log x=0$.

Corollary 3 Let $\boldsymbol{q}=\left(q_{1}, \ldots, q_{k}\right)$ and $\boldsymbol{r}=\left(r_{1}, \ldots, r_{k}\right)$ be positive real $\boldsymbol{k}$-tuples, and $\boldsymbol{w}=$ $\left(w_{1}, \ldots, w_{k}\right)$ be a positive probability distribution such that conditions (45) and (46) hold with $\frac{q_{i}}{w_{i}}, \frac{r_{i}}{w_{i}} \in\left[\vartheta_{1}, \vartheta_{2}\right](i=1,2, \ldots, k)$. If $\log$ has base $b$ greater than 1 and $\frac{q}{w}$ and $\frac{r}{w}$ are decreasing, then for the Shannon entropy of $\boldsymbol{w}$, we have the following estimate:

$$
\begin{aligned}
H(\boldsymbol{w}) \leq & \sum_{i=1}^{k} w_{i} \log \left(\frac{r_{i}}{w_{i}}\right)-\sum_{s=2}^{n-1}\left(\frac{n-s}{(s-2) !\left(\vartheta_{2}-\vartheta_{1}\right)}\right) \int_{\vartheta_{1}}^{\vartheta_{2}}\left(\widetilde{I}_{G_{l}}(\boldsymbol{q}, \boldsymbol{w}, v)-\widetilde{I}_{G_{l}}(\boldsymbol{r}, \boldsymbol{w}, v)\right) \\
& \times\left(\frac{(-1)^{s-1}(s-2) !}{\vartheta_{2}^{s-1} \ln b}\left(v-\vartheta_{2}\right)^{s-2}-\frac{(-1)^{s-1}(s-2) !}{\vartheta_{1}^{s-1} \ln b}\left(v-\vartheta_{1}\right)^{s-2}\right) d v .
\end{aligned}
$$

If $\log$ has base b between 0 and 1 , then inequality (49) is reversed.

Proof Take $f(x):=-\log x$, which is an $n$-convex function for even $n>3$ and $q_{i}=1(i=$ $1,2, \ldots, k)$. Then using Theorem 6 , we get (49). Moreover, for odd $n>3$, inequality (49) is reversed.

Corollary 4 Let $\boldsymbol{q}=\left(q_{1}, \ldots, q_{k}\right)$ and $\boldsymbol{r}=\left(r_{1}, \ldots, r_{k}\right)$ be positive probability distributions such that conditions (45) and (46) hold with $q_{i}, r_{i} \in\left[\vartheta_{1}, \vartheta_{2}\right](i=1,2, \ldots, k)$. If $\log$ has base $b$ greater than 1 and $\boldsymbol{q}$ and $\boldsymbol{r}$ are decreasing, then the relation between the Shannon entropies of $\boldsymbol{q}$ and $\boldsymbol{r}$ is given by the following estimate:

$$
\begin{aligned}
H(\boldsymbol{q}) \leq & H(\boldsymbol{r})-\sum_{s=2}^{n-1}\left(\frac{n-s}{(s-2) !\left(\vartheta_{2}-\vartheta_{1}\right)}\right) \int_{\vartheta_{1}}^{\vartheta_{2}}\left(\widetilde{I}_{G_{l}}(\boldsymbol{q}, \boldsymbol{w}, v)-\widetilde{I}_{G_{l}}(\boldsymbol{r}, \boldsymbol{w}, v)\right) \\
& \times\left((x \log x)^{(s-1)}\left(\vartheta_{2}\right)\left(v-\vartheta_{2}\right)^{s-2}-(x \log x)^{(s-1)}\left(\vartheta_{1}\right)\left(v-\vartheta_{1}\right)^{s-2}\right) d v,
\end{aligned}
$$

where for $d=1,2,(x \log x)^{\prime}\left(\vartheta_{d}\right)=\frac{1}{\ln b}\left(1+\ln \vartheta_{d}\right)$ and $(x \log x)^{(s-1)}\left(\vartheta_{d}\right)=\frac{(-1)^{s-1}(s-3) !}{\vartheta_{d}^{s-2} \ln b}, s \geq 3$. If $\log$ has base $b$ between 0 and 1 , then inequality (50) is reversed.

Proof Take $g(x):=\log x$, so that $x g(x):=x \log x$ is an $n$-convex function for even $n>3$ and $w_{i}=1(i=1,2, \ldots, k)$. Then by Theorem 7 we get (50). Moreover, for odd $n>3$, inequality (50) is reversed.

In the second case, we study generalized majorization-type inequality in terms of the K-L distance or relative entropy between two probability distributions. 
Definition 5 Let $\mathbf{r}=\left(r_{1}, \ldots, r_{k}\right)$ and $\mathbf{w}=\left(w_{1}, \ldots, w_{k}\right)$ be two positive probability distributions. Then the K-L distance between them is defined by

$$
L(\mathbf{r}, \mathbf{w}):=\sum_{i=1}^{k} r_{i} \log \left(\frac{r_{i}}{w_{i}}\right) .
$$

Corollary 5 Let $\boldsymbol{q}=\left(q_{1}, \ldots, q_{k}\right), \boldsymbol{r}=\left(r_{1}, \ldots, r_{k}\right)$, and $\boldsymbol{w}=\left(w_{1}, \ldots, w_{k}\right)$ be positive real $k$-tuples such that conditions (45) and (46) hold with $\frac{q_{i}}{w_{i}}, \frac{r_{i}}{w_{i}} \in\left[\vartheta_{1}, \vartheta_{2}\right](i=1,2, \ldots, k)$. If $\log$ has base $b$ greater than 1 and $\frac{q}{w}$ and $\frac{r}{w}$ are decreasing, then

$$
\begin{aligned}
\sum_{i=1}^{k} w_{i} \log \left(\frac{q_{i}}{w_{i}}\right) \leq & \sum_{i=1}^{k} w_{i} \log \left(\frac{r_{i}}{w_{i}}\right)-\sum_{s=2}^{n-1}\left(\frac{n-s}{(s-2) !\left(\vartheta_{2}-\vartheta_{1}\right)}\right) \\
& \times \int_{\vartheta_{1}}^{\vartheta_{2}}\left(\widetilde{I}_{G_{l}}(\boldsymbol{q}, \boldsymbol{w}, v)-\widetilde{I}_{G_{l}}(\boldsymbol{r}, \boldsymbol{w}, v)\right) \\
& \times\left(\frac{(-1)^{s-1}(s-2) !}{\ln b\left(v-\vartheta_{2}\right)^{2-s} \vartheta_{2}^{s-1}}-\frac{(-1)^{s-1}(s-2) !}{\ln b\left(v-\vartheta_{1}\right)^{2-s} \vartheta_{1}^{s-1}}\right) d v .
\end{aligned}
$$

If $\log$ has base b between 0 and 1 , then inequality (51) is reversed.

Proof Take $f(x):=-\log x$, which is an $n$-convex function for $n>3$. Then by Theorem 6 we get (51). Moreover, for odd $n>3$, inequality (51) is reversed.

Corollary 6 Let $\boldsymbol{q}=\left(q_{1}, \ldots, q_{k}\right), \boldsymbol{r}=\left(r_{1}, \ldots, r_{k}\right)$, and $\boldsymbol{w}=\left(w_{1}, \ldots, w_{k}\right)$ be positive probability distributions such that conditions (45) and (46) hold with $\frac{q_{i}}{w_{i}}, \frac{r_{i}}{w_{i}} \in\left[\vartheta_{1}, \vartheta_{2}\right](i=1,2, \ldots, k)$. If $\log$ has base $b$ greater than 1 and $\frac{q}{w}$ and $\frac{r}{w}$ are decreasing, then the relation between the $K-L$ distance of $(\boldsymbol{r}, \boldsymbol{w})$ and $(\boldsymbol{q}, \boldsymbol{w})$ is given by the following estimate:

$$
\begin{aligned}
L(\boldsymbol{q}, \boldsymbol{w}) \geq & L(\boldsymbol{r}, \boldsymbol{w})+\sum_{s=2}^{n-1}\left(\frac{n-s}{(s-2) !\left(\vartheta_{2}-\vartheta_{1}\right)}\right) \int_{\vartheta_{1}}^{\vartheta_{2}}\left(\widetilde{I}_{G_{l}}(\boldsymbol{q}, \boldsymbol{w}, v)-\widetilde{I}_{G_{l}}(\boldsymbol{r}, \boldsymbol{w}, v)\right) \\
& \times\left((x \log x)^{(s-1)}\left(\vartheta_{2}\right)\left(v-\vartheta_{2}\right)^{s-2}-(x \log x)^{(s-1)}\left(\vartheta_{1}\right)\left(v-\vartheta_{1}\right)^{s-2}\right) d v,
\end{aligned}
$$

where for $d=1,2,(x \log x)^{\prime}\left(\vartheta_{d}\right)=\frac{1}{\ln b}\left(1+\ln \vartheta_{d}\right)$ and $(x \log x)^{(s-1)}\left(\vartheta_{d}\right)=\frac{(-1)^{s-1}(s-3) !}{\vartheta_{d}^{s-2} \ln b}, s \geq 3$. If $\log$ has base $b$ between 0 and 1 , then inequality (52) is reversed.

Proof Take $g(x):=\log x$, so that $x g(x):=x \log x$ is an $n$-convex function for even $n>3$. Then by Theorem 7 we get (52). Moreover, for odd $n>3$, inequality (52) is reversed.

\section{Acknowledgements}

The authors are thankful to the anonymous referees for reading the manuscript and giving fruitful comments and suggestions. The Ministry of Education and Science of Russian Federation has supported the research of the fourth author (the Agreement number No. 02.a03.21.0008).

Funding

There is no funding for this work.

Availability of data and materials

Data sharing is not applicable to this paper as no datasets were generated or analyzed during the current study. 
Authors' contributions

All the authors contributed equally in this research. All authors read and approved the final manuscript.

\section{Author details}

'Department of Mathematics, Government College University, 38000 Faisalabad, Pakistan. ${ }^{2}$ Department of Mathematics, University of Sargodha, 40100 Sargodha, Pakistan. ${ }^{3}$ Rudn University, Moscow, Russia.

\section{Publisher's Note}

Springer Nature remains neutral with regard to jurisdictional claims in published maps and institutional affiliations.

Received: 30 March 2020 Accepted: 8 July 2020 Published online: 22 July 2020

\section{References}

1. Adeel, M., Khan, K.A., Pečarić, Đ., Pečarić, J.: Generalization of the Levinson inequality with applications to information theory. J. Inequal. Appl. 2019, 230 (2019)

2. Adeel, M., Khan, K.A., Pečarić, Đ., Pečarić, J.: Levinson type inequalities for higher order convex functions via Abel-Gontscharoff interpolation. Adv. Differ. Equ. 2019, 430 (2019)

3. Adeel, M., Khan, K.A., Pečarić, Đ., Pečarić, J.: Estimation of $f$-divergence and Shannon entropy by Levinson type inequalities via new Green's functions and Lidstone polynomial. Adv. Differ. Equ. 2020, 27 (2020)

4. Adeel, M., Khan, K.A., Pečarić, Đ., Pečarić, J.: Estimation of $f$-divergence and Shannon entropy by using Levinson type inequalities for higher order convex functions via Hermite interpolating polynomial. J. Inequal. Appl. 2020, 137 (2020)

5. Adeel, M., Khan, K.A., Pečarić, Đ., Pečarić, J.: Estimation of $f$-divergence and Shannon entropy by Levinson type inequalities for higher order convex functions via Taylor polynomial. J. Math. Comput. Sci. 21(4), 322-334 (2020)

6. Agarwal, R.P., Wong, P.J.Y.: Error Inequalities in Polynomial Interpolation and Their Applications. Kluwer Academic, Dordrecht (1993)

7. Alberti, P.M., Uhlmann, A.: Stochasticity and partial order: doubly stochastic maps and unitary mixing Dordrecht, Boston (1982)

8. Ando, T.: Majorization, doubly stochastic matrices, and comparison of eigenvalues. Linear Algebra Appl. 118, 163-248 (1989)

9. Ando, T.: Majorizations and inequalities in matrix theory. Linear Algebra Appl. 199, 17-67 (1994)

10. Bernett, N.S., Cerone, P., Dragomir, S.S.: Majorization inequalities for Stieltjes integrals. Appl. Math. Lett. 22, 416-421 (2009)

11. Csiszár, l.: Information-type measures of differences of probability distributions and indirect observations. Studia Sci. Math. Hung. 2, 299-318 (1967)

12. Csiszár, l.: Information measures: a critical survey. In: Trans. 7th Prague Conf. on Info. Th., Statist. Decis. Funct., Random Processes and 8th European Meeting of Statist., Volume B, pp. 73-86. Academia, Praque (1978)

13. Fink, A.M.: Bounds of the deviation of a function from its averages. Czechoslov. Math. J. 42(117), $289-310$ (1992)

14. Fuchs, L.: A new proof of an inequality of Hardy-Littlewood-Pólya. Math. Tidsskr. B, 53-54 (1947)

15. Hardy, G.H., Littlewood, J.E., Pólya, G.: Inequalities. Cambridge University Press, Cambridge (1978)

16. Horváth, L., Pečarić, $Đ$., Pečarić, J.: Estimations of $f$-and Rényi divergences by using a cyclic refinement of the Jensen's inequality. Bull. Malays. Math. Sci. Soc. 2018(1), 318 (2018)

17. Jakšetić, J., Pečarić, J.: Exponential convexity method. J. Convex Anal. 20(1), 181-197 (2013)

18. Jakšetić, J., Pečarić, J., Perušić, A.: Steffensen inequality, higher order convexity and exponential convexity. Rend. Circ. Mat. Palermo 63(1), 109-127 (2014)

19. Khalid, S., Pečarić, J., Perić, I.: Refinements and generalizations of majorization, Favard and Berwald-type inequalities via Fink identity. J. Math. Inequal. 12, 267-284 (2018)

20. Khan, M.A., Bradanović, S.I., Latif, N., Pečarić, Đ., Pečarić, J.: Majorization Inequality and Information Theory. Element, Zagreb (2019)

21. Khan, M.A., Khalid, S., Pečarić, J.: Refinements of some majorization type inequalities. J. Math. Inequal. 7(1), 73-92 (2013)

22. Khan, M.A., Latif, N., Pečarić, J.: Generalization of majorization theorem. J. Math. Inequal. 9(3), 847-872 (2015)

23. Khan, M.A., Latif, N., Perić, I., Pečarić, J.: On Sapogov's extension of Čebyšev's inequality. Thai J. Math. 10(2), 617-633 (2012)

24. Khan, M.A., Latif, N., Perić, I., Pečarić, J.: On majorization for matrices. Math. Balk. 27 (2013), Fasc. 1-2

25. Khan, M.A., Niezgoda, M., Pečarić, J.: On a refinement of the majorization type inequality. Demonstr. Math. 44(1), 49-57 (2011)

26. Latif, N., Pečarić, J., Perić, I.: On majorization, Favard's and Berwald's inequalities. Ann. Funct. Anal. 2(1), 31-50 (2011)

27. Latif, N., Siddique, N., Pečarić, J.: Generalization of majorization theorem-II. J. Math. Inequal. 12(3), 731-752 (2018)

28. Maligranda, L., Pečarić, J., Persson, L.E.: Weighted Favard's and Berwald's inequalities. J. Math. Anal. Appl. 190, 248-262 (1995)

29. Marshall, A.W., Olkin, I., Arnold, B.C.: Inequalities: Theory of Majorization and Its Applications, 2nd edn. Springer Series in Statistics. Springer, New York (2011)

30. Mehmood, N., Agarwal, R.P., Butt, S.I., Pečarić, J.: New generalization of Popoviciu type inequalities via new Green functions and Montgomery identity. J. Inequal. Appl. 2017, 108 (2017)

31. Pečarić, J.: On some inequalities for functions with nondecreasing increments. J. Math. Anal. Appl. 98, 188-197 (1984)

32. Pečarić, J., Perić, J.: Improvement of the Giaccardi and the Petrović inequality and related Stolarsky type means. An. Univ. Craiova, Ser. Mat. Inform. 39(1), 65-75 (2012)

33. Pečarić, J., Proschan, F., Tong, Y.L.: Convex Functions, Partial Orderings and Statistical Applications. Academic Press, New York (1992)

34. Siddique, N., Latif, N., Pečarić, J.: Generalized results of majorization inequality via Lidstone's polynomial and newly Green functions. J. Nonlinear Sci. Appl. 11(6), 812-831 (2018) 